\title{
A light scalar WIMP through the Higgs portal and CoGeNT
}

\author{
Sarah Andreas, ${ }^{1}$ Chiara Arina, ${ }^{2}$ Thomas Hambye, ${ }^{2}$ Fu-Sin Ling,,${ }^{2,3}$ and Michel H.G. Tytgat ${ }^{2}$ \\ ${ }^{1}$ Deutsches Elektronen Synchrotron, Notkestrasse 85, D-22603 Hamburg, Germany \\ ${ }^{2}$ Service de Physique Théorique, Université Libre de Bruxelles, \\ CP225, Bld du Triomphe, 1050 Brussels, Belgium \\ ${ }^{3}$ Institute for Particle Physics Phenomenology, Durham University, DH1 3LE, UK
}

\begin{abstract}
If dark matter (DM) simply consists in a scalar particle interacting dominantly with the Higgs boson, the ratio of its annihilation cross section - which is relevant both for the relic abundance and indirect detection - and its spin-independent scattering cross section on nuclei depends only on the DM mass. It is an intriguing result that, fixing the mass and direct detection rate to fit the annual modulation observed by the DAMA experiment, one obtains a relic density in perfect agreement with its observed value. In this short letter we update this result and confront the model to the recent CoGeNT data, tentatively interpreting the excess of events in the recoil energy spectrum as being due to DM. CoGeNT, as DAMA, points toward a light DM candidate, with somewhat different (but not necessarily incompatible) masses and cross sections. For the CoGeNT region too, we find an intriguing agreement between the scalar DM relic density and direct detection constraints. We also give the one $\sigma$ region favoured by the CDMS-II events, that suggest a light DM candidate too, and the limits from Xenon10 2009 data, which, depending on the assumed scintillation efficiency, may exclude both CoGeNT and DAMA. Assuming CoGeNT and/or DAMA to be due to DM leads to definite predictions regarding indirect detection and Higgs search at the LHC.
\end{abstract}

Recently, there has been some effervescence regarding what may be first hints of direct detection of dark matter (DM) from the galactic halo. The most recent is related to the CoGeNT experiment, a low background germanium crystal based detector, with a rather modest exposure time and detector mass, but very low threshold energy, which has announced an anomaly in the form of an excess of events at low recoil energies 11. Although the collaboration clearly leans toward natural radioactivity as the cause for the observed excess, they do put forward the possibility that the events may be explained by the elastic collisions of DM from the galactic halo, with a mass in the $\sim 7-10$ $\mathrm{GeV}$ range, and a rather large spin independent (SI) cross section on nuclei, $\sigma_{S I} \sim 7 \times 10^{-41} \mathrm{~cm}^{2}$.

Surprizingly, these values for the mass and scattering cross section are not too different from those required to fit the DAMA/Libra and DAMA/NaI (DAMA in the sequel) events. DAMA has observed 11 successive cycles of annual modulation in the rate of nuclear recoils, with a statistical significance of $8.2 \sigma[2]$. These measurements are consistent with the signal that would arise from elastic scattering of a WIMP from the galactic halo with the nuclei in the detectors, the flux of DM particles being modulated by the periodic motion of the Earth around the Sun [3, 4].

There has been much work on the DM interpretation of the recent DAMA data (see e.g. [5 20]). In [21] (see also [22]), it has been shown that the DAMA results may be explained as being caused by the elastic scattering of a singlet scalar DM candidate interacting through the Higgs portal. This particle may be a true singlet scalar [23] 25], or the low energy limit of another model ${ }^{1}$. In the present short letter, we provide an update on the status of the singlet scalar in the light of the recent data from the CoGeNT collaboration, tentatively interpreted as being due to DM. We also include the recent data of the CMDS-II collaboration [30. With 2 events for an expected background of $0.8 \pm 0.2$ events, the significance of this result is low, but it also hints at a rather light DM candidate.

We adopt the convention of [21, and consider just one real singlet scalar $S$, together with a $Z_{2}$ symmetry, $S \rightarrow-S$, to ensure its stability, so that the following renormalizable terms may be added to the SM lagrangian:

$$
\mathcal{L} \ni \frac{1}{2} \partial^{\mu} S \partial_{\mu} S-\frac{1}{2} \mu_{S}^{2} S^{2}-\frac{\lambda_{S}}{4} S^{4}-\lambda_{L} H^{\dagger} H S^{2}
$$

where $H$ is the Standard Model Higgs doublet, and the mass of $S$ is given by

$$
m_{S}^{2}=\mu_{S}^{2}+\lambda_{L} \mathrm{v}^{2}
$$

with $\mathrm{v}=246 \mathrm{GeV}$. Both annihilation into SM particles and scattering with nuclei are through the coupling $\lambda_{L}$ to the Higgs particle $h$, respectively in the s and t-channel. Annihilation through the Higgs is S-wave, and the cross section for scattering on nuclei is purely of the Spin Independent (SI) type. For a DM candidate lighter than the Higgs,

\footnotetext{
1 A possible implementation is the Inert Doublet Model (IDM), which is another very simple extension of the Standard Model with scalar DM [26-29].
} 


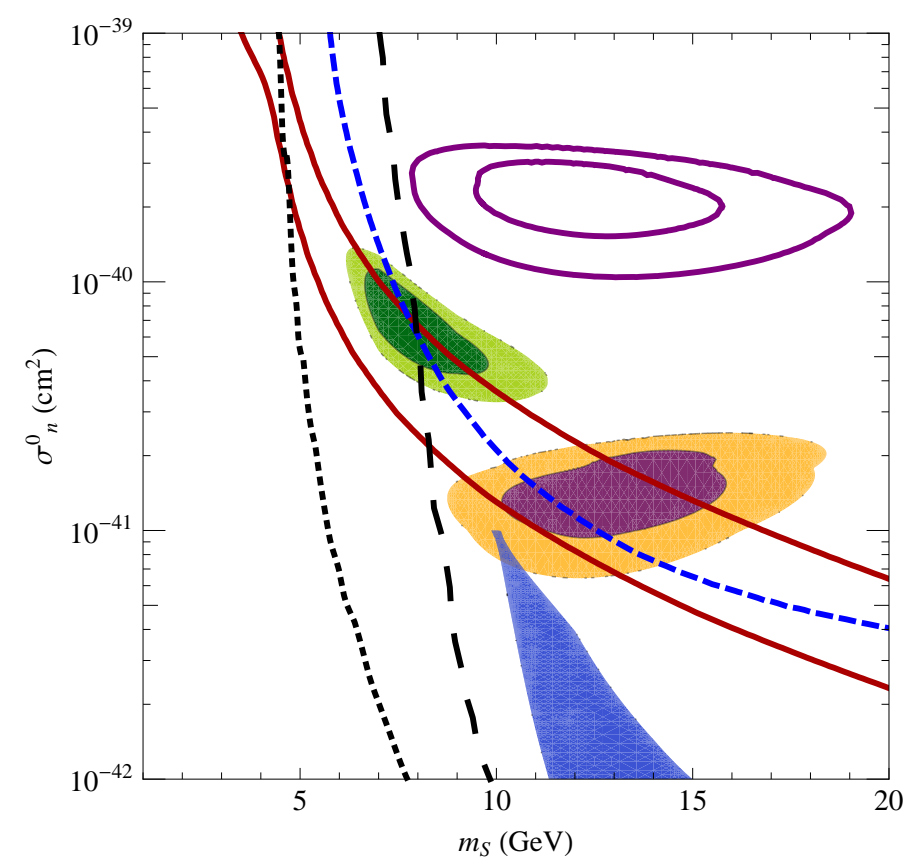

Figure 1: SI cross section $\left(\sigma_{n}^{0}\right)$ vs scalar singlet mass $\left(m_{S}\right)$, for $\rho_{D M}=0.3 \mathrm{GeV} / \mathrm{cm}^{3}$ and a standard Maxwellian velocity distribution (with mean velocity $220 \mathrm{~km} / \mathrm{s}$ and escape velocity $v_{e s c}=650 \mathrm{~km} / \mathrm{s}$, see our conventions in 22 ). The green region corresponds to CoGeNT (minimum $\chi^{2}$, with contours at 90 and $99.9 \%$ C.L.), for which we have assumed that the excess at low recoil energies is entirely due to DM (assuming a constant background contamination). The DAMA regions (goodness-of-fit, also at 90 and $99.9 \%$ C.L.) are given both with (purple/orange) and without (purple, no fill) channelling. The blue region corresponds to the CDMS-II two events, at $1 \sigma$, which we obtained following the procedure of [36. The blue (short-dashed) line is the $90 \%$ C.L. exclusion limit from CDMS-Si [37. The black dotted line is the 90\% C.L. exclusion limit from the Xenon10 2009 data set, using their scintillation efficiency [38, as also considered in 36. The long-dashed line is based on the same data but using instead the smaller scintillation efficiency advocated in [39] (central value, at $1 \sigma$ the corresponding exclusion can be found in 34). Finally, the brown lines (continuous) encompass the region predicted by the singlet scalar DM model corresponding to the WMAP range $0.094 \leq \Omega_{D M} h^{2} \leq 0.129$, for $0.2 \leq f \leq 0.4$.

$m_{S} \ll m_{h}$, the ratio of the annihilation and scattering cross section depends only on $m_{S}$,

$$
\sum_{\mathrm{f}} \frac{\sigma(S S \rightarrow \overline{\mathrm{ff}}) v_{r e l}}{\sigma(S N \rightarrow S N)}=\sum_{\mathrm{f}} \frac{n_{c} m_{\mathrm{f}}^{2}}{f^{2} m_{N}^{2} \mu_{r}^{2}} \frac{\left(m_{S}^{2}-m_{\mathrm{f}}^{2}\right)^{3 / 2}}{m_{S}}
$$

where $n_{c}=3(1)$ for quarks (leptons), and $\mu_{r}=m_{S} m_{N} /\left(m_{S}+m_{N}\right)$ is the nucleon-DM reduced mass. The factor $f$ parametrizes the Higgs to nucleons coupling, $f m_{N} \equiv\left\langle N\left|\sum_{q} m_{q} \bar{q} q\right| N\right\rangle=g_{h N N} \mathrm{v}$, and we consider $0.2 \leq f \leq 0.4$ (see e.g. [31).

Equation (3) shows that the mass of the DM candidate is fixed for a given relic abundance and SI scattering cross section [21, 23 25. In turn, direct detection experiments may determine both the SI cross section and the mass of the DM, modulo the astrophysical uncertainties regarding the local density and velocity distribution of the DM. A priori there is little chance that these constraints may be met by singlet scalar DM, but as Figure 1 reveals, the model may be in agreement with CoGeNT — which is the main result of this letter - or, as shown in [21, 22], with DAMA. Since there is a gap between the CoGeNT and DAMA (with channelling) regions, that scalar DM agrees with CoGeNT does not trivially derive from the fact that it may agree with DAMA. We emphasize that this result, as for DAMA, is specific to a scalar particle with scalar couplings to SM fermions. For instance, annihilation through the Higgs portal would be P-wave suppressed for a fermionic singlet DM candidate, and other interactions, as is the case for a light neutralino [32, are necessary to agree with the direct detection data (see also e.g. 333 35]).

The gap between CoGeNT and DAMA may be reduced, either assuming that channelling is less effective than what is advocated by the DAMA collaboration (which has the effect of raising the DAMA region - but not reducing the tension with exclusion limits), or assuming that the CoGeNT excess is partially contaminated by some natural radioactivity (lowering the CoGeNT region) or a mixture of both. One may also adjust the properties of the halo or the DAMA spectral data [34, but we have refrained from doing so. 

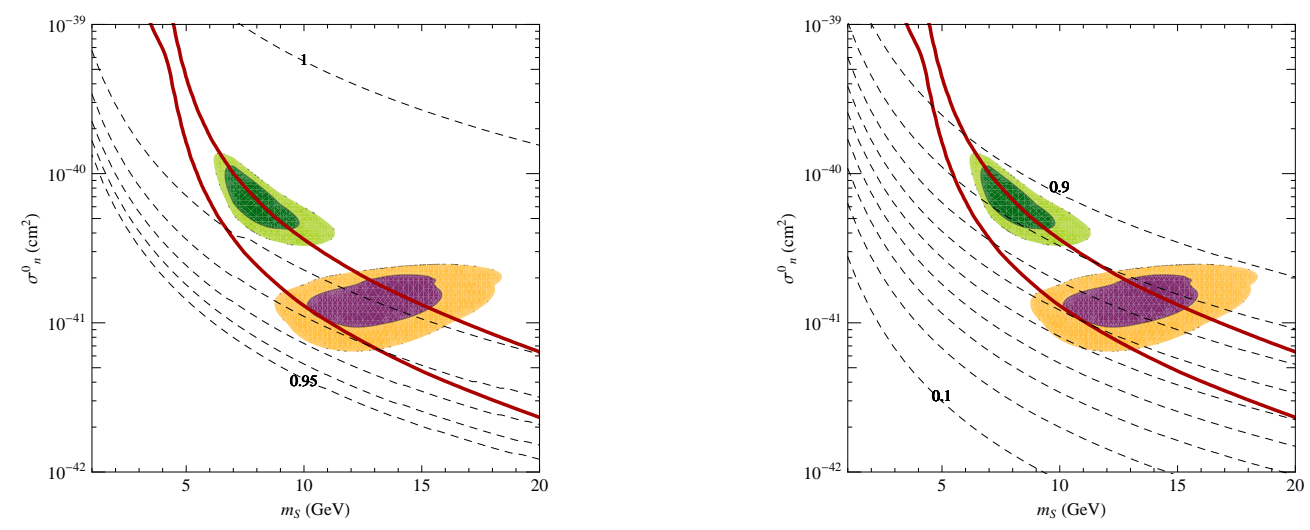

Figure 2: Higgs invisible decay branching ratio for $m_{h}=120 \mathrm{GeV}$ (left panel) and $m_{h}=180 \mathrm{GeV}$ (right panel).

Also, both regions are excluded by the most stringent limit set by Xenon10 (the dotted line in the figure). However, if the scintillation efficiency - the measure of the fraction of energy from the recoiling nuclei that goes into scintillation light - is actually lower than that used by the collaboration (the long-dashed line in the figure), as advocated in [39], there is a region of CoGeNT which may be consistent with all experimental constraints (and the singlet scalar DM candidate).

In the scenario we consider, the coupling $\lambda_{L}$ to the Higgs must be fairly large to explain both the relic abundance ${ }^{2}$ and the direct detection data. For $m_{h}=120 \mathrm{GeV}\left(m_{h}=180 \mathrm{GeV}\right)$, one typically requires $\lambda_{L} \simeq-0.2$ (resp. $\left.\simeq-0.45\right)$ for a $\mathrm{DM}$ candidate of mass $m_{S} \sim 8 \mathrm{GeV}$. For the same choice of parameters, $\mu_{S} \sim 100 \mathrm{GeV}$, which implies fine tuning of the parameters at the level of the percent, which is not unbearable in our opinion. Also there is no mechanism to naturally stabilize the mass of the scalar at a scale of few GeV. Note however that, if neither CoGeNT nor DAMA could be fitted with $\mu_{S}^{2} \sim 0$ at tree level, in the Inert Doublet Model at one loop, the DM mass and coupling ranges required by CoGeNT and/or DAMA may be compatible with dynamical electroweak symmetry breaking induced by the inert doublet [42].

A light scalar dark matter candidate coupled to the Higgs has potentially many other signatures or implications, a large flux of gamma rays from dark matter annihilations [10, 21], a large flux of neutrinos from capture by the Sun, which may be constrained by Super-Kamiokande [13, 43 45], or anti-protons and anti-deuterons in cosmic rays [5, 46]. Its annihilation cross section and mass make it also a very natural candidate to solve the ${ }^{6} \mathrm{Li}$ problem (see the Figure 3 in [47]).

Last but not least, a light WIMP in the form of a scalar coupled to the Higgs would imply that the Higgs mostly decays into a pair of dark matter particles $[21,24,25$. We would like to point out that the DAMA and CoGeNT regions could be distinguished from a measurement of the invisible Higgs decay branching ratio. For $m_{h}=180 \mathrm{GeV}$ the effect is striking: as Figure 2 shows, taking into account the CDMS-Si limit and the WMAP region, the DAMA region gives $60 \% \lesssim B R \lesssim 70 \%$, while for the CoGeNT region, one has $75 \% \lesssim B R \lesssim 90 \%$. This difference is larger than the expected $\sim 10 \%$ LHC sensitivity on the invisible branching ratio 48 . For $m_{h}=120 \mathrm{GeV}$, the difference is much reduced, because the invisible channel largely dominates the decay width: we get $98 \% \lesssim B R \lesssim 99 \%$ and $B R \gtrsim 99 \%$ for DAMA and CoGeNT respectively.

\footnotetext{
2 In the calculation of the relic abundance, we neglect the possible effect of the QCD phase transition, which is supposed to have taken place around $\sim 150 \mathrm{MeV}$ (see, for instance, [40]). Since typically $x_{f}=m_{D M} / T_{f o} \simeq 20$, for $m_{S} \simeq 6 \mathrm{GeV}$, for instance, the freeze-out temperature is $T_{f o} \simeq 300 \mathrm{MeV} \gg 150 \mathrm{MeV}$ and thus the QCD phase transition is irrelevant for the range of mass we consider. Otherwise, the QCD phase transition might increase the relic abundance by at most a factor of $\mathrm{O}(2)$ (see for instance 41]). Such an increase could be compensated by an increase of $\lambda_{L}$ by a factor of $\sim 1.4$ and this, in turn, would require a decrease of the parameter $f$ by the same factor.
} 


\section{Acknowledgments}

We thank Thomas Schwetz and Karsten Jedamzik for useful discussions. Our work is supported by the FNRS-FRS, the IISN and the Belgian Science Policy (IAP VI-11).

[1] CoGeNT, C. E. Aalseth et al., (2010), 1002.4703.

[2] DAMA, R. Bernabei et al., Eur. Phys. J. C56, 333 (2008), arXiv:0804.2741.

[3] A. K. Drukier, K. Freese, and D. N. Spergel, Phys. Rev. D33, 3495 (1986).

[4] K. Freese, J. A. Frieman, and A. Gould, Phys. Rev. D37, 3388 (1988).

[5] A. Bottino, F. Donato, N. Fornengo, and S. Scopel, Phys. Rev. D78, 083520 (2008), arXiv:0806.4099.

[6] R. Foot, Phys. Rev. D78, 043529 (2008), 0804.4518.

[7] S. Chang, A. Pierce, and N. Weiner, (2008), arXiv:0808.0196.

[8] M. Y. Khlopov, (2008), 0806.3581.

[9] DAMA, R. Bernabei et al., Mod. Phys. Lett. A23, 2125 (2008), 0802.4336.

[10] J. L. Feng, J. Kumar, and L. E. Strigari, Phys. Lett. B670, 37 (2008), 0806.3746.

[11] E. Masso, S. Mohanty, and S. Rao, (2009), 0906.1979.

[12] F. Petriello and K. M. Zurek, JHEP 09, 047 (2008), arXiv:0806.3989.

[13] C. Savage, G. Gelmini, P. Gondolo, and K. Freese, (2008), arXiv:0808.3607.

[14] S. Chang, G. D. Kribs, D. Tucker-Smith, and N. Weiner, (2008), arXiv:0807.2250.

[15] E. Dudas, S. Lavignac, and J. Parmentier, Nucl. Phys. B808, 237 (2009), 0808.0562.

[16] Y. Cui, D. E. Morrissey, D. Poland, and L. Randall, (2009), arXiv:0901.0557.

[17] J. March-Russell, C. McCabe, and M. McCullough, (2008), arXiv:0812.1931.

[18] M. Fairbairn and T. Schwetz, JCAP 0901, 037 (2009), arXiv:0808.0704.

[19] A. Bandyopadhyay, S. Chakraborty, A. Ghosal, and D. Majumdar, (2010), 1003.0809.

[20] F.-S. Ling, (2009), 0911.2321.

[21] S. Andreas, T. Hambye, and M. H. G. Tytgat, JCAP 0810, 034 (2008), arXiv:0808.0255.

[22] C. Arina, F.-S. Ling, and M. H. G. Tytgat, JCAP 0910, 018 (2009), 0907.0430.

[23] J. McDonald, Phys. Rev. D50, 3637 (1994), hep-ph/0702143.

[24] C. P. Burgess, M. Pospelov, and T. ter Veldhuis, Nucl. Phys. B619, 709 (2001), hep-ph/0011335.

[25] V. Barger, P. Langacker, M. McCaskey, M. J. Ramsey-Musolf, and G. Shaughnessy, Phys. Rev. D77, 035005 (2008), 0706.4311.

[26] N. G. Deshpande and E. Ma, Phys. Rev. D18, 2574 (1978).

[27] R. Barbieri, L. J. Hall, and V. S. Rychkov, Phys. Rev. D74, 015007 (2006), hep-ph/0603188.

[28] E. Ma, Phys. Rev. D73, 077301 (2006), hep-ph/0601225.

[29] L. Lopez Honorez, E. Nezri, J. F. Oliver, and M. H. G. Tytgat, JCAP 0702, 028 (2007), hep-ph/0612275.

[30] The CDMS-II, Z. Ahmed et al., (2009), 0912.3592.

[31] J. Gasser, H. Leutwyler, and M. E. Sainio, Phys. Lett. B253, 260 (1991).

[32] A. Bottino, F. Donato, N. Fornengo, and S. Scopel, Phys. Rev. D77, 015002 (2008), arXiv:0710.0553.

[33] Y. G. Kim and S. Shin, JHEP 05, 036 (2009), 0901.2609.

[34] A. L. Fitzpatrick, D. Hooper, and K. M. Zurek, (2010), 1003.0014.

[35] E. Kuflik, A. Pierce, and K. M. Zurek, (2010), 1003.0682.

[36] J. Kopp, T. Schwetz, and J. Zupan, (2009), 0912.4264.

[37] CDMS, D. S. Akerib et al., Phys. Rev. Lett. 96, 011302 (2006), astro-ph/0509259.

[38] XENON10, J. Angle et al., Phys. Rev. D80, 115005 (2009), 0910.3698.

[39] A. Manzur et al., (2009), 0909.1063.

[40] L. McLerran, (2008), 0808.1057.

[41] A. Bottino, F. Donato, N. Fornengo, and S. Scopel, Phys. Rev. D68, 043506 (2003), hep-ph/0304080.

[42] T. Hambye and M. H. G. Tytgat, Phys. Lett. B659, 651 (2008), 0707.0633.

[43] C. Savage, K. Freese, P. Gondolo, and D. Spolyar, (2009), arXiv:0901.2713.

[44] S. Andreas, M. H. G. Tytgat, and Q. Swillens, JCAP 0904, 004 (2009), 0901.1750.

[45] J. L. Feng, J. Kumar, J. Learned, and L. E. Strigari, (2008), 0808.4151.

[46] E. Nezri, M. H. G. Tytgat, and G. Vertongen, JCAP 0904, 014 (2009), 0901.2556.

[47] K. Jedamzik and M. Pospelov, New J. Phys. 11, 105028 (2009), 0906.2087.

[48] O. J. P. Eboli and D. Zeppenfeld, Phys. Lett. B495, 147 (2000), hep-ph/0009158. 